Classification

Physics Abstracts

$77.80 \mathrm{c}-64.70 \mathrm{p}-78.30 \mathrm{j}$

\title{
Displacive-order-disorder crossover at the ferroelectric-paraelectric phase transitions of $\mathrm{BaTiO}_{3}$ and $\mathrm{LiTaO}_{3}$
}

\author{
K. A. Müller \\ IBM Zurich Research Laboratory, 8803 Rüschlikon, Switzerland \\ Y. Luspin (*), J. L. Servoin and F. Gervais \\ Centre de Recherches sur la Physique des Hautes Températures, \\ Centre National de la Recherche Scientifique, 45045 Orléans, France
}

(Reçu le 12 février 1982, révisé le 22 avril, accepté le 4 mai 1982)

\begin{abstract}
Résumé. - On compare les données reçues de mesures précises de réflectivité infrarouge et de constantes diélectriques $\varepsilon_{0}(T)$ dans certains ferroélectriques $\mathrm{ABO}_{3}$. Dans $\mathrm{BaTiO}_{3}$, le mode ferroélectrique se sature au-dessus de la transition cubique tétragonale tandis que $\varepsilon_{0}(T)$ tend à diverger. Ceci s'interprète en terme de changement de régime dominant, passant de displacif à ordre-désordre à l'approche de $T_{\mathrm{c}}$, en accord avec la dimensionalité critique dans les systèmes cubiques dipolaires $d_{\mathrm{c}}=4>d=3$. Dans le composé uniaxe $\mathrm{LiTaO}_{3}$ où $d=d_{\mathrm{c}}=3$, le phénomène est en effet moins marqué.
\end{abstract}

\begin{abstract}
A detailed comparison between accurate infrared reflectivity data and dielectric constant $\varepsilon_{0}(T)$ measurements has been carried out in $\mathrm{ABO}_{3}$ ferroelectrics. In $\mathrm{BaTiO}_{3}$, the ferroelectric mode saturates above the cubic-tetragonal transition, whereas $\varepsilon_{0}(T)$ tends to diverge. This supports an intrinsic crossover from displacive to order-disorder behaviour on approaching $T_{\mathrm{c}}$ because the critical dimensionality in these cubic dipolar systems is $d_{\mathrm{c}}=4>d=3$. In the uniaxial $\mathrm{LiTaO}_{3}$ with $d=d_{\mathrm{c}}=3$, the crossover is less pronounced.
\end{abstract}

From computer simulations [1, 2], as well as analytic theory [3], a new view regarding the behaviour of displacive continuous structural phase transitions (SPT) near $T_{\mathrm{c}}$ has emerged : the crossover from a regime in which the collective behaviour has classical displacive soft-mode behaviour to a regime whose features are better described in the language traditionally reserved for order-disorder systems [4]. The present work indicates this to occur in the important class of ferroelectric perovskite systems, and thus points towards a resolution of the long-standing controversy between the soft-mode versus order-disorder description in $\mathrm{BaTiO}_{3}$ and $\mathrm{KNbO}_{3}$ as well as in $\mathrm{LiTaO}_{3}$. The soft-mode behaviour was supported by early infrared reflectivity measurements [5] and later inelastic neutron-scattering data [6]. The order-disorder picture stemmed from the observation of strong diffusive X-ray scattering [7], as well as Raman activity above the tetra-

(*) Permanent address : Laboratoires d'Etudes Physiques des Matériaux, Université d'Orléans, 45045 Orléans, France. 
gonal-cubic transition [8] and more recent Raman investigations in the tetragonal phase [9]. The situation turned out to be similarly controversial in $\mathrm{LiTaO}_{3}[10]$.

The displacive to order-disorder regime is due to intrinsic anharmonic behaviour from correlated fluctuations near wave vector $\mathbf{q}=0$ (in space and time). One expects from renormalizationgroup (R.G.) theory that correlated fluctuations near $T_{\mathrm{c}}$ are more pronounced the larger $\varepsilon=d_{\mathrm{c}}-d$ is, $d_{\mathrm{c}}$ being the critical dimensionality of a system [11]. The antiferrodistortive SPT in $\mathrm{SrTiO}_{3}$ has $d_{\mathrm{c}}=4, \dot{d}=3$, i.e., $\varepsilon=1$, and experimental results established quantitatively precursor order [12]. In $\mathrm{KH}_{2} \mathrm{PO}_{4}, d_{\mathrm{c}}=2.5$ due to soft-mode/acoustic-mode coupling. With $d=3, \varepsilon=-0.5$ one expects Gaussian mean-field behaviour. It has been shown [13] that in $\mathrm{KH}_{2} \mathrm{PO}_{4}$, the statics and dynamics follow quantitatively mean-field behaviour. Courtens was the first to suggest [13a] the possible importance of the critical dimensionality $d_{c}$ in the question of the occurrence of intrinsic central peaks.

Uniaxial ferroelectrics like TGS with their long-range dipolar interactions have a critical dimensionality $d_{\mathrm{c}}=3$. In this case critical behaviour is given by logarithmic corrections to classical Landau results [14]. Thus, fluctuation effects are already present but not dominating. Isotropic dipolar systems with dipolar and short-range interactions have been investigated using R.G. theory [15]. The static critical behaviour calculated is nearly indistinguishable from that in cubic short-range systems $[11,15]$ and $d_{\mathrm{c}}=4$. In real cubic oxide ferroelectrics, cubic anisotropic interactions are also present. Their fixed-point behaviour depends on the cubic anisotropy of the soft-modes. The fixed-point behaviour is either isotropic dipolar, not stable $\left(\mathrm{BaTiO}_{3}\right.$ ?) or cubic dipolar [16]. Kind and Müller [17] measured the exponent of the dielectric susceptibility $\varepsilon_{0}$ above the second-order phase transition in a cubic $\mathrm{KTa}_{0.9} \mathrm{Nb}_{0.1} \mathrm{O}_{3}$ crystal to be $\gamma=1.7$ [17], well outside the mean-field behaviour of $\gamma=1$ [11]. Thus, one might expect that for cubic ferroelectrics like $\mathrm{BaTiO}_{3}$ and $\mathrm{KNbO}_{3}$, a crossover from displacive to order-disorder behaviour may exist on approaching $T_{\mathrm{c}}^{+}$. Infrared reflectivity measurements [18] show this occurs as described below.

The new soft-mode data have been obtained using a Fourier-transform scanning interferometer, Bruker IFS113. These modern i.r. spectrometers are extremely well-suited to measure infrared active modes accurately in the frequency range from 10 to $4000 \mathrm{~cm}^{-1}$ and up to high temperatures $(1300 \mathrm{~K})$. The infrared reflectivity data were analysed on the basis of a factorized form of the dielectric function [18]. Typical experimental data and an example of data fits are shown in figure $1 a$. Simulations of infrared reflectivity, restricted for simplicity to the soft-mode alone and inserted in figures $1 b$ and $c$, show that changes of TO soft-mode frequency (Fig. $1 b$ ) and linewidth (Fig. 1c) typical of oxidic perovskites give rise to quite significant changes of reflectivity profiles. In fact, infrared reflectivity yields information on the real part of the dielectric function in addition to the imaginary part. Only the latter is accessible by scattering (Raman or neutrons). Soft-mode frequency parameters, in particular, may be accurately deduced from low-frequency reflectivity which appears slightly dependent on damping as shown in the left-hand insert of figure la even when the soft mode is overdamped. Results at room temperature agree with those of [5]. Both data sets disagree with Ballantyne's spectrum [19] at room temperature. Results at other temperatures were previously compared [18] to other spectral data in detail and found to be in agreement with most of the data. In particular, extrapolating neutron data of [6] to $q=0$ appears compatible with present soft-mode frequencies.

Results for the soft-mode in $\mathrm{BaTiO}_{3}$ in the cubic paraelectric and tetragonal ferroelectric phases are given in figure $2 a$. The curves show a clear-cut soft-mode behaviour over a wide range of temperatures, whose extrapolation intersects the temperature axis near $T_{\mathrm{c}}$. However, on approaching $T_{\mathrm{c}}$, $\Omega_{\mathrm{To}}$ starts to level some $120 \mathrm{~K}$ above the phase transition to a value of $60 \mathrm{~cm}^{-1}$. Recent hyperRaman measurements also confirm the saturation over that temperature range [20]. At $T_{\mathrm{c}}$, an $A_{1}$-type component is abruptly shifted to $\approx 280 \mathrm{~cm}^{-1}$, consistent with the first-order transition, while an E-component continues to soften to the next tetragonal-to-orthorhombic transition. Thus, the E-mode shows no significant discontinuity. Similar behaviour has been observed in the 


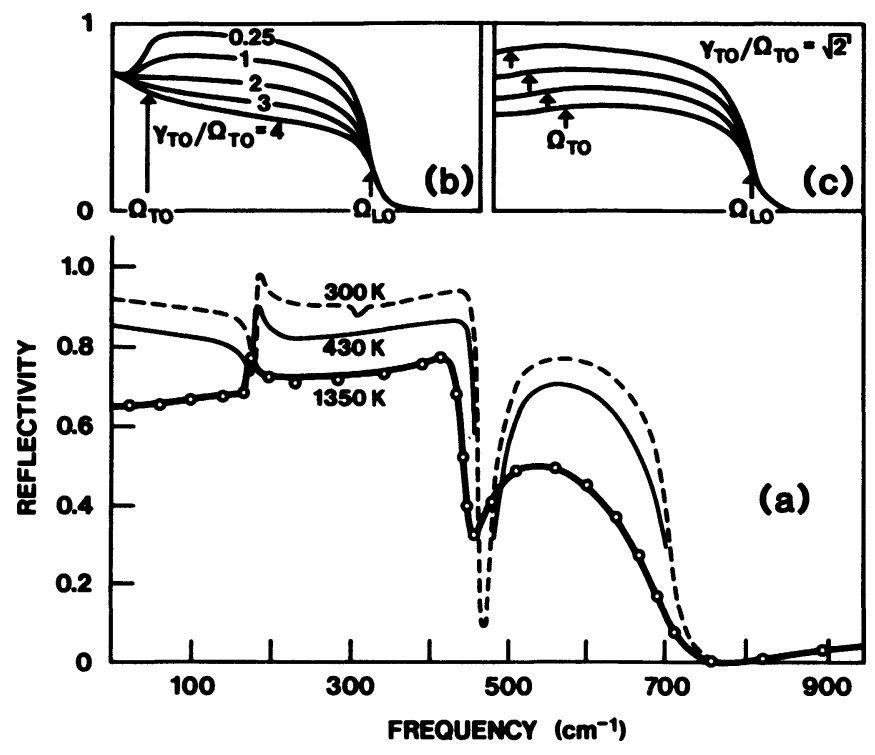

Fig. 1. - (a) Typical reflectivity profiles in $\mathrm{BaTiO}_{3}$ at several temperatures. Best fit (heavy full curve) to experimental data $(O)$ at $1350 \mathrm{~K}$, for example. See text for inserts $(b)$ and $(c)$.

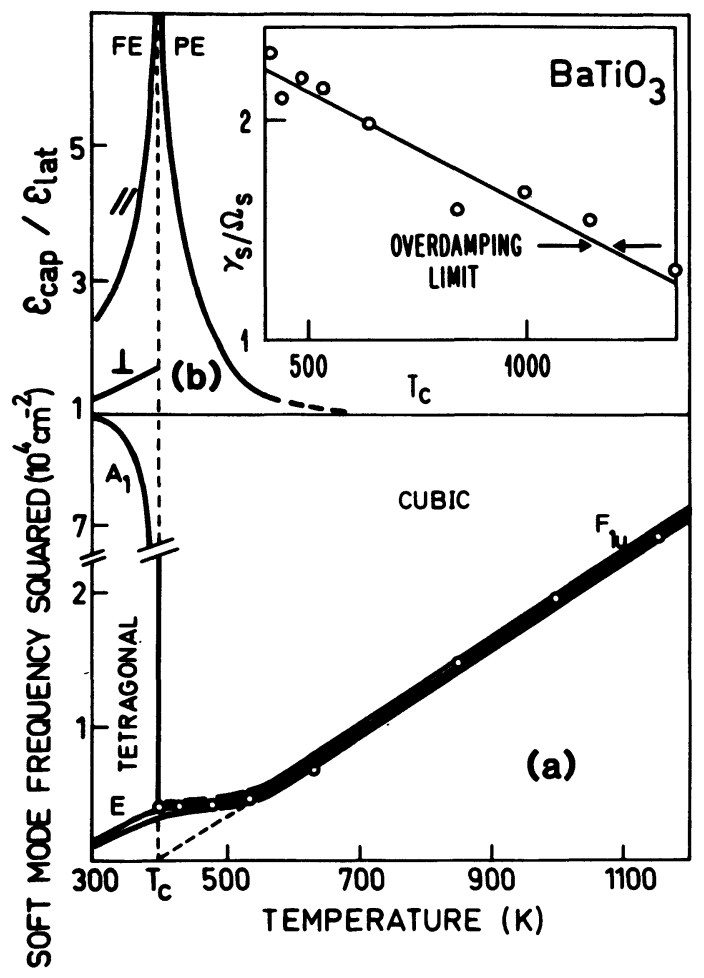

Fig. 2. - Temperature dependence of the three ferroelectric soft-mode components of $\mathrm{BaTiO}_{3}(a)$, and the ratio of the " static " over the dielectric constant calculated from lattice modes $(b)$ via $\varepsilon_{1 \text { at }}=\varepsilon_{\infty} \prod_{j} \Omega_{j \mathrm{jO}}^{2} / \Omega_{\text {jTO}}^{2}$. Note that the $\Omega_{j}$ 's are moduli of complex poles (or zeroes). Insert shows passage from underdamped to overdamped soft-mode behaviour. 
isomorphous phase of $\mathrm{KNbO}_{3}$ [21]. Furthermore, in both crystals the soft-mode damping is heavy, over ten times that of the next modes, and persists into the lower tetragonal phase (see figure 2 insert).

The ratio of the dielectric constants $\varepsilon_{\text {cap }} / \varepsilon_{\text {lat }}$, with $\varepsilon_{\text {cap }}$ measured in the $\mathrm{MHz}$ range, above piezoelectric resonances, and $\varepsilon_{\text {lat }}$ calculated from the lattice modes shows the following (Fig. $\left.2 b\right):$ the soft-mode roughly explains $\varepsilon_{\text {cap }}$ at sufficiently high temperatures (Fig. 1a) but this becomes less and less true on approaching $T_{\mathrm{c}}$ from above [22]. Thus, a dynamical disorder affects the displacive behaviour and an additional relaxation can be related to disorder [7]. Therefore, we arrive at the conclusion that a displacive-to-order-disorder crossover occurs as has been shown to be present in the antiferrodistortive SPT of $\mathrm{SrTiO}_{3}$ [12]. The latter transition is second-order, and deviations from mean-field behaviour appear $10 \%$ in $t=\left(T-T_{\mathrm{c}}\right) / T_{\mathrm{c}}$ below $T_{\mathrm{c}}=T_{0}$ for the rotational order parameter, and above $T_{\mathrm{c}}$ for the soft-mode as measured by inelastic neutrons [23]. In the present case of $\mathrm{BaTiO}_{3}$, the deviation in the soft-mode $\Omega_{\text {To }}$ appears $25 \%$ above the $T_{0}$, where the mean-field extrapolated $\Omega_{\text {To }}$ vanishes. This is thus comparable to the magnitude found for the short-range $\mathrm{SrTiO}_{3}$ system. In our opinion, these new findings settle the controversy whether the cubic-to-tetragonal transitions in $\mathrm{BaTiO}_{3}$ and $\mathrm{KNbO}_{3}$ are displacive or orderdisorder.

It should be noted that in figure 2 , the high-temperature ratio $\varepsilon_{\text {cap }} / \varepsilon_{\text {lat }}$ has its low-temperature counterpart reflected about $T_{\mathrm{c}}$, as is expected from scaling for a continuous transition, figure $2 a$, but the soft mode has none, figure $2 b$, i.e., the $A_{1}$ component has jumped to $280 \mathrm{~cm}^{-1}$ and therefore cannot be responsible for the $\varepsilon_{\text {cap }}$ enhancement towards $T_{\mathrm{c}}^{-}$in any way [22] ! Thus close to $T_{\mathrm{c}}$ an additional time-dependent dynamic motion distinct from the soft-mode behaviour has to be present. In fact, a relaxation of the dielectric constant, along the FE axis has been observed recently near 3-4 GHz at room temperature [24], the high-frequency value being in agreement with

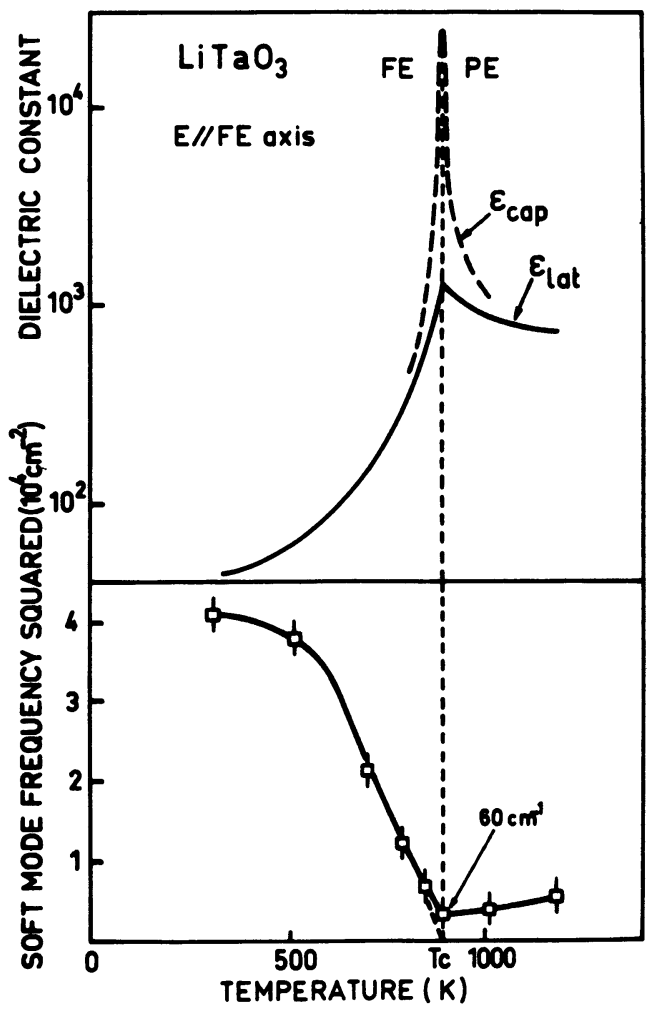

Fig. 3. - Temperature dependence of the soft-mode frequency of $\mathrm{LiTaO}_{3}$ (lower part), and comparison of $\varepsilon_{\text {lat }}$ with the « static » dielectric constant (upper part-note logarithmic scale). 
$\varepsilon_{\text {lat }}$, while no marked relaxation of $\varepsilon_{\perp}$ was found perpendicular to the $\mathrm{FE}$ axis above piezoelectric resonances, consistent with the results of figure 2 . Thus, the vibrational motion of the mobile ion with respect to its neighbours is very anharmonic and should exhibit a central-peak response near $T_{\mathrm{c}}$, in addition to the saturating soft-mode at $\sim 60 \mathrm{~cm}^{-1}$. It has to be a relaxational motion, which might be slowed-down further by coupling to slowly relaxing impurities. The computer simulations of Schneider and Stoll [25] show that the dynamics are slowed-down by a constant factor but are otherwise unaltered in their critical behaviour. Most recent index of refraction measurements for $T>T_{\mathrm{c}}$ in flux and melt-grown $\mathrm{BaTiO}_{3}$ crystals have been interpreted in terms of intrinsic dynamic islands of polarization [26].

The uniaxial ferroelectric $\mathrm{LiTaO}_{3}$ has also been investigated with the same method. $\Omega_{\text {soft }}$ again does not freeze-out, and $\varepsilon_{\text {cap }} / \varepsilon_{\text {lat }}$ increases on approaching $T_{\mathrm{c}}$ but the range in temperature is more restricted to the immediate vicinity of the transition (Fig. 3). Thus, the behaviour is all in all more " classical ». This is, however, expected in view of the fact that for this uniaxial crystal $d=d_{\mathrm{c}}=3$, i.e., the dimensionality is marginal, and correlated fluctuations for $\mathbf{q}=\mathbf{0}$ are present but dominate over a narrower $T / T_{\mathrm{c}}$ temperature range than in $\mathrm{BaTiO}_{3}$.

In summary, the soft-mode behaviour in $\mathrm{ABO}_{3}$ ferroelectrics, especially the cubic ones, saturates well above the ferroelectric transition. A comparison with the measured dielectric constants indicates a crossover from displacive to order-disorder behaviour on approaching $T_{\mathrm{c}}$. This is in agreement with expectations of precursor order from the renormalization-group point of view. The present findings give indirect evidence that cubic ferroelectrics do not behave classically as has always been assumed except for one experiment [17] on a second-order cubic-to-tetragonal ferroelectric transition.

We have benefited from correspondence with H.D. Bruce and G. Burns.

\section{References}

[1] Schneider, T. and Stoll, E., Phys. Rev. Lett. 31 (1973) 1254 ; Phys. Rev. B 13 (1976) 1216.

[2] Aubry, S., J. Chem. Phys. 62 (1975) 3217; 64 (1976) 3392 and

Koehler, T. R., Bishop, A. R., Krumhansl, J. A. and Schrieffer, J. R., Solid State Commun. 17 (1975) 1515.

[3] Krumhansl, J. A. and Schrieffer, J. R., Phys. Rev. B 11 (1975) 3535.

[4] BRuCE, A. D., in Solitons and Condensed-Matter Physics, editors A. R. Bishop and T. Schneider (Springer-Verlag, Heidelberg) 1978, p. 116.

[5] Spitzer, W. G., Miller, R. C., Kleinman, D. A. and Howarth, L. E., Phys. Rev. 126 (1962) 1710 ; Barker, A. S., Phys. Rev. 145 (1966) 391.

[6] Harada, J., Axe, J. D. and Shirane, G., Phys. Rev. B 4 (1971) 155.

[7] Comes, R., LAMBert, M. and Guinier, A., Solid State Commun. 6 (1968) 715 ;

LAMBert, M. and Comes, R., Solid State Commun. 7 (1969) 305.

[8] QuitTET, A. M. and LAMBert, M., Solid State Commun. 12 (1973) 1053.

[9] Heiman, D. and Ushioda, S., Phys. Rev. B 9 (1974) 2122 ;

Burns, G. and Dacot, F. H., Phys. Rev. B 18 (1978) 5750.

[10] Abrahams, S. C., Buehler, E., Hamilton, W. C. and Laplaca, S. J., J. Phys. Chem. Solids 34 (1973) 521 ;

Penna, A. F., Porto, S. P. S. and Chaves, A. S., in Light Scattering in Solids, editors M. Balkanski, R. C. C. Leite and S. P. S. Porto (Flammarion, Paris) 1976, p. 890.

[11] Fischer, M. E., Rev. Mod. Phys. 46 (1974) 597.

[12] Bruce, A. D., Mueller, K. A. and Berlinger, W., Phys. Rev. Lett. 42 (1979) 185.

[13] a) Courtens, E., Phys. Rev. Lett. 41 (1978) 1171 ;

b) Courtens, E., Gammon, R. and Alexander, S., Phys. Rev. Lett. 43 (1979) 1026 ; and

c) to be published.

[14] Larkin, A. I. and Khmel' nitskil, D. E., Sov. Phys.-JETP 29 (1969) 1123 ;

Strukov, B. A., Ferroelectrics 12 (1976) 97 ;

EHSES, K. H. and MUESER, H. E., Ferroelectrics 12 (1976) 247. 
[15] Aharony, A. and Fisher, M. E., Phys. Rev. B 8 (1973) 3323 ;

Bruce, A. D. and Aharony, A., Phys. Rev. B 10 (1974) 2078.

[16] Verstraeten, G., Z. Phys. B-Condensed Matter 43 (1981) 149.

[17] Kind, R. and Mueller, K. A., Commun. Phys. 1 (1976) 223 ;

Bilz, H., Migoni, R., Meissner, G. and Mueller, K. A., EMF-4, Portoroz, Yugoslavia, 1979.

[18] Luspin, Y., Servoin, J. L. and Gervais, F., J. Phys. C 13 (1980) 3761.

[19] Ballantyne, J. M., Phys. Rev. 136 (1964) 429.

[20] Inoue, K. and Asat, N., to appear in J. Physique.

[21] Quittet, A. M., Servoin, J. L. and Gervais, F., J. Physique 42 (1981) 493 ;

Fontana, M., Metrat, G., Servoin, J. L. and Gervais, F., to be published.

[22] Burns, G. and BursteIn, E., Ferroelectrics 7 (1974) 297 ;

Halperin, B. I. and Varma, C. M., Phys. Rev. B 14 (1976) 4030. The theory of « dirty " displacive ferroelectrics, i.e., containing impurities which couple to the ferroelectric mode, cannot account for this behaviour : while the dielectric-constant ratio clearly has divergent character towards $T_{\mathrm{c}}$, the soft-mode frequency is nearly constant.

[23] Currat, R., Mueller, K. A., Berlinger, W. and Denoyer, F., Phys. Rev. B 17 (1978) 2937.

[24] Turik, A. V. and Shevchenko, N. B., Phys. Status Solidi B 95 (1979) 585.

[25] Schneider, T. and Stoll, E., Phys. Rev. B 16 (1977) 2220.

[26] BuRNs, G. and DACOL, F. H., to appear in Ferroelectrics. 